\title{
ON THE ASPHERICITY OF RIBBON DISC COMPLEMENTS
}

BY

JAMES HOWIE ${ }^{1}$

\begin{abstract}
The complement of a ribbon $n$-disc in the $(n+2)$-ball has a 2 -dimensional spine which shares some of the combinatorial properties of classical knot complement spines. It is an open question whether such 2-complexes are always aspherical. To any ribbon disc we associate a labelled oriented tree, from which the homotopy type of the complement can be recovered, and we prove asphericity in certain special cases described by conditions on this tree. Our main result is that the complement is aspherical whenever the associated tree has diameter at most 3.
\end{abstract}

1. Introduction. There has been considerable interest recently in higher dimensional ribbon knots and ribbon discs $[3,6,9,12]$. A ribbon $n$-disc $(n \geqslant 2)$ is a proper embedding $k: D^{n} \hookrightarrow D^{n+2}$, such that $r \circ k: D^{n} \rightarrow \mathbf{R}$ is a Morse function with no critical points of index more than 1 , where $r: D^{n+2} \rightarrow \mathbf{R}$ is the radial function $x \mapsto\|x\|$. A ribbon $n$-knot is the boundary $k: S^{n} \hookrightarrow S^{n+2}$ of a ribbon $(n+1)$-disc $k$ : $D^{n+1} \hookrightarrow D^{n+3}$.

In this paper we will be concerned with the question of whether a ribbon disc complement $D^{n+2}-k\left(D^{n}\right)$ is necessarily aspherical. There are various motivations for studying this question. First, the asphericity of ribbon disc complements is an important special case of the "Whitehead Conjecture" that subcomplexes of aspherical 2-complexes are aspherical $[3,12]$. Second, ribbon disc complements have 2-dimensional spines which are combinatorially very similar to spines of classical knot complements in $S^{3}$, which is an indication that they may well be aspherical. Moreover, a proof of their asphericity would probably entail a direct proof of the asphericity of classical knot complements, independent of (although presumably more difficult than) powerful 3-manifold machinery such as the sphere theorem. Third, the homotopy type of higher ribbon knot complements was shown in [3] to be of a particularly simple form, provided ribbon disc complements are aspherical. Unfortunately, the proof offered in [3] for the asphericity of ribbon disc complements is incomplete. Nor is [3] the only place in the literature in which an incomplete proof of this assertion is given (see [12] for a discussion), so that the problem would appear to be a subtle one.

Received by the editors June 18, 1984.

1980 Mathematics Subject Classification. Primary 57M20; Secondary 20E25, 20F05, 57Q45.

Key nords and phrases. Ribbon discs, aspherical 2-complexes, locally indicable groups.

${ }^{1}$ Supported by an SERC Advanced Fellowship. 
Any given ribbon disc $k: D^{n} \hookrightarrow D^{n+2}$ can be pushed out to the boundary $S^{n+1}$ of $D^{n+2}$ to give an immersion $i: D^{n} \rightarrow S^{n+1}$ whose only singularities are a finite number of disjoint codimension 1 discs of double points. Furthermore, the inverse image in $D^{n}$ of each such double $(n-1)$-disc consists of one small $D^{n-1}$ in the interior of $D^{n}$, together with one properly embedded $D^{n-1}$ (that is, $D^{n-1} \cap \partial D^{n}=$ $\left.\partial D^{n-1}\right)$, and the properly embedded $(n-1)$-discs separate $D^{n}$ into finitely many subdiscs (see [9] for details in the case $n=2$; the general case is similar). Each double $(n-1)$-disc of this form is called a ribbon intersection, and an immersion whose only singularities are ribbon intersections will be called a ribbon immersion. Conversely, any ribbon immersion $D^{n} \rightarrow \rightarrow S^{n+1}$ can be pushed slightly into $D^{n+2}$ to obtain a ribbon disc. Thus an $(n-1)$-knot is ribbon if and only if it bounds a ribbon immersion $D^{n} \rightarrow S^{n+1}$.

We shall prove asphericity of ribbon discs in certain special cases. In order to state our main results, consider a ribbon disc $k: D^{n} \hookrightarrow D^{n+2}$ with associated ribbon immersion $i: D^{n} \rightarrow S^{n+1}$. Let $\Sigma$ denote the singular set of $i$, and define a pseudometric $\mu$ on $X=D^{n}-i^{-1}(\Sigma)$ as follows. For $x, y \in X$, let $\mu(x, y)$ be the least number of points of $i^{-1}(\Sigma)$ on any path in $D^{n}$ from $x$ to $y$. This number is finite by transversality, and $\mu$ is clearly an integer-valued pseudometric on $X$.

THEOREM A. If $(X, \mu)$ has diameter at most 3 , then $D^{n+2}-k\left(D^{n}\right)$ is aspherical.

Here diameter of $(X, \mu)$ means $\sup \{\mu(x, y) ; x, y \in X\}$. The diameter condition is fairly stringent, and yet the proof of Theorem $\mathrm{A}$ is depressingly complicated. It works by a detailed analysis of the 2-complex spine of the complement of $k$, and in fact it yields the following slightly stronger result.

THEOREM B. Under the hypotheses of Theorem A, $\pi_{1}\left(D^{n+2}-k\left(D^{n}\right)\right)$ is locally indicable.

A group is locally indicable if each of its nontrivial, finitely generated subgroups admits an infinite cyclic homomorphic image. Theorem A follows immediately from Theorem B, using the fact that disc complements are homology circles, together with known properties of locally indicable groups (see $§ 5$, in particular Fact 5.1).

Now it is not difficult to see that the universal quotient metric space of $(X, \mu)$ consists of the vertex set of a certain finite tree $\Gamma$, equipped with the edge-path length metric. The tree $\Gamma$, referred to as the dual graph of the ribbon immersion $i$ : $D^{n} \rightarrow S^{n+1}$, is discussed in detail in $\$ 2$. It comes naturally equipped with an orientation and a "labelling" of its edges. With this extra structure $\Gamma$ completely determines the homotopy type of $D^{n+2}-k\left(D^{n}\right)$ (but not the isotopy type of $k$ ).

In \$3 such labelled oriented trees (or, more generally, graphs) are discussed in the abstract. To each oriented labelled graph $\Gamma$ is associated a presentation $P(\Gamma)$ of a group $G(\Gamma)$, and hence a 2-complex $K(\Gamma)$ with $\pi_{1} K(\Gamma) \cong G(\Gamma)$. In the case where $\Gamma$ is the dual graph of a ribbon immersion $i: D^{n} \rightarrow S^{n+1}, K(\Gamma)$ is a spine of the corresponding ribbon disc complement $D^{n+2}-k\left(D^{n}\right)$. Furthermore, for each integer $n \geqslant 2$, any labelled oriented tree can be realised as the dual graph of a ribbon immersion $D^{n} \rightarrow \rightarrow S^{n-1}$. 
Now the dual graph of a ribbon immersion corresponds to a decomposition of that immersion into subdiscs connected by ribbons, which is more specialised than the decompositions normally considered (see, for example, $[3,9])$. The more general situation corresponds to a finite tree with a "weak labelling" which takes values in a free group. Such "weakly labelled oriented trees" are discussed in §4. Again, to each weakly labelled oriented tree $\Gamma$ is associated a presentation $P(\Gamma)$ of a group $G(\Gamma)$, and so a 2-complex $K(\Gamma)$. Various moves on weakly labelled oriented trees are described, which induce extended Nielsen transformations on the presentations, and so 3-deformations on the 2-complexes.

In $\$ 5$ we recall some basic facts about locally indicable groups, and about "reducibility" in presentations and 2-complexes, in the sense of [11]. These are the main tools which we will use later in the paper.

The groundwork for the proof of Theorem B is contained in $\$ \S 6$ and 7, and the proof is completed in $\S 8$. In $\S 6$ the concept of a "sloping word" in a free group is introduced, and the following is proved.

THEOREM 6.2. Let $\Gamma$ be a weakly labelled oriented tree with 3 vertices, in which at least one of the labels is a reduced sloping word. Then $G(\Gamma)$ is locally indicable.

If $\Gamma$ is a weakly labelled oriented tree with 2 vertices, then $G(\Gamma)$ is a torsion free 1-relator group, so locally indicable by [4]. The group $G(\Gamma)$ in Theorem 6.2 is a 2-relator group in which the defining relators are of a very special kind, and it is reasonable to conjecture that the result holds even without the sloping word condition. I know no way of proving this, but fortunately Theorem 6.2 is sufficient for our purposes as it stands.

In $\$ 7$ the notions of admissible subgraph and generating set of vertices are introduced. Two technical results are proved (Theorem 7.1 and Corollary 7.2), which are aimed at reducing the study of a given weakly labelled oriented tree to that of a smaller one (possibly with longer labels).

In $\S 8$ we apply the machinery of $\S 7$ to the case of a labelled oriented tree of diameter at most 3 . The outcome is a weakly labelled oriented tree with at most 3 vertices and reduced sloping words for labels. We then apply Theorem 6.2 to complete the proof of Theorem B (and hence also of Theorem A).

Theorem $B$ is the best result of a general nature which we can prove with the methods of this paper. Nevertheless these methods are sufficiently powerful to prove asphericity in many special cases not covered by Theorem A. In \$§9-11 we give some examples of such results.

In $\$ 9$ we give some conditions on labelled oriented trees of diameter 4 under which the proof of Theorem B (with some minor modifications) still works.

In $\$ 10$ we associate three further graphs $I(\Gamma), T(\Gamma)$ and $U(\Gamma)$ to a labelled oriented graph $\Gamma$. If $\Gamma$ and one of $I(\Gamma), T(\Gamma)$ are trees, then $G(\Gamma)$ is (locally free)-by-(infinite cyclic), while if all three are trees, then $G(\Gamma)$ is free-by-(infinite cyclic) (Theorem 10.1). A condition on $U(\Gamma)$ translates to a small cancellation condition on $P(\Gamma)$, and so shows that $K(\Gamma)$ is aspherical (Theorem 10.3). This is the only one of our results in which we prove asphericity without proving local 
indicability. This is not surprising, since it uses small cancellation theory, and there exist finitely generated perfect small cancellation groups (see for example [15]). On the other hand, this result applies whether or not $\Gamma$ is a tree. Furthermore, the small cancellation condition also ensures that $P(\Gamma)$ has soluble word and conjugacy problems.

In $\$ 11$ we consider the following class of 2-relator group presentations. Let $F$ be the free group on three generators $x, y, z$, and let $f: F \rightarrow F$ be the endomorphism $x \mapsto x^{2} y x^{-1} y^{-1}, y \mapsto y^{2} z y^{-1} z^{-1}, z \mapsto z^{2} x z^{-1} x^{-1}$. Let $G_{n}$ be the group given by the presentation

$$
P_{n}:\left\langle x, y, z \mid f^{n}(x), f^{n}(y)\right\rangle
$$

where $f^{n}$ denotes the $n$th iterate of $f$. This section was motivated by a question put to me some time ago by Martin Dunwoody: is $P_{2}$ aspherical?

In fact we show that $G_{n}$ is locally indicable, whence $P_{n}$ is aspherical for all $n \geqslant 0$. It is not too difficult to show that $P_{n}$ is equivalent under extended Nielsen transformations to $P(\Gamma)$ for some labelled oriented tree $\Gamma$ (of large diameter). Hence we have found another large class of ribbon disc complements to be asphercial.

2. The dual graph of a ribbon immersion. Let $i: D^{n} \rightarrow S^{n+1}$ be a ribbon immersion, and let $\alpha_{1}, \ldots, \alpha_{m}$ be the double $(n-1)$-discs of $i$ in $S^{n+1}$ (Figure 1). The inverse image of $\alpha_{j}$ consists of a properly embedded $(n-1)$-disc $\gamma_{j}$ in $D^{n}$, meeting $\partial D^{n}$ precisely in its boundary, together with a small $(n-1)$-disc $\gamma_{j}^{\prime}$ contained entirely in Int $D^{n}$ (Figure 2). We will refer to the $\gamma_{j}$ as separating discs. Ignoring the nonseparating discs $\gamma_{j}^{\prime}$ for the moment, consider the decomposition $\left(D^{n} ; \gamma_{1}, \ldots, \gamma_{m}\right)$ of $D^{n}$ given by the separating discs. Dual to this decomposition is a graph $\Gamma$. Explicitly, $\Gamma$ has a vertex for each component of $D^{n}-\left(\gamma_{1} \cup \cdots \cup \gamma_{m}\right)$ and an edge for each $\gamma_{j}$. The edge corresponding to $\gamma_{j}$ joins the vertices corresponding to the two components separated by $\gamma_{j}$. (Compare the "graph of a surface" defined in [18].)

Since $D^{n}$ is simply connected, it follows that $\Gamma$ is a tree. Furthermore, two points $x$ and $y$ of $D^{n}-\left(\gamma_{1} \cup \cdots \cup \gamma_{m} \cup \gamma_{1}^{\prime} \cup \cdots \cup \gamma_{m}^{\prime}\right)$ lie in the same (resp. adjacent) component(s) of $D^{n}-\left(\gamma_{1} \cup \cdots \cup \gamma_{m}\right)$ if and only if $\mu(x, y)=0$ (resp. 1). It follows that the universal quotient metric space of $(X, \mu)$ is isometric to the vertex set $V(\Gamma)$ with the edge-path length metric. In particular, the diameter of the pseudometric space $(X, \mu)$ is just the diameter of the tree $\Gamma$.

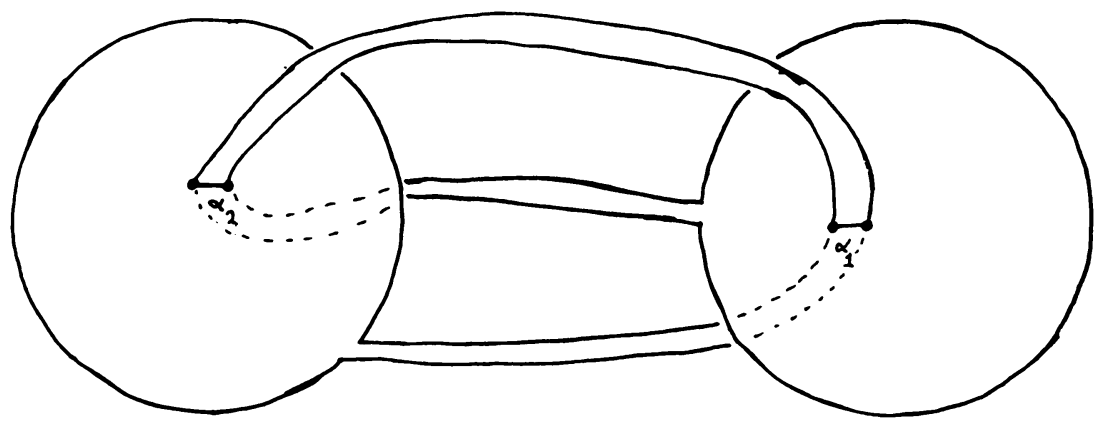

FIGURE 1 


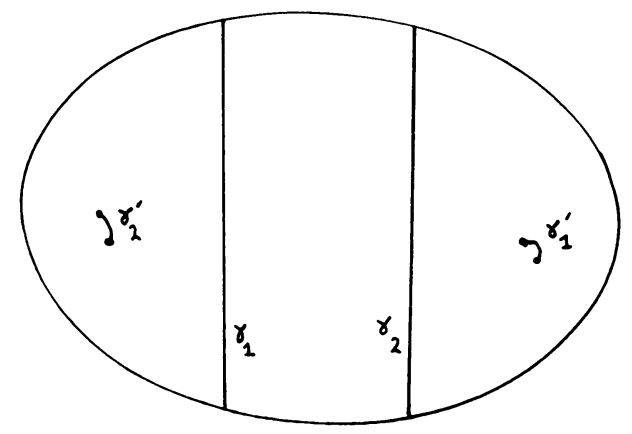

FIGURE 2

Note also that $\Gamma$ can be nicely embedded in $D^{n}$ so that each vertex lies in the corresponding component of $D^{n}-\left(\gamma_{1} \cup \cdots \cup \gamma_{m}\right)$, and each edge intersects the corresponding separating disc $\gamma_{j}$ transversely in a single point, and such that $\Gamma$ is disjoint from the $\gamma_{j}$ and $\gamma_{j}^{\prime}$ apart from these transverse crossings (Figure 3).

There are two pieces of additional structure which we can naturally associate to the tree $\Gamma$. The first is an orientation. There is a well-defined positive normal direction to $i\left(D^{n}\right)$ in $S^{n+1}$ induced by the standard orientations of $D^{n}$ and $S^{n+1}$. At a double $(n-1)$-disc $\alpha_{j}$ we may assume that the two sheets intersect normally, so that the positive normal to the sheet containing $\gamma_{j}^{\prime}$ pulls back to a choice of normal direction to $\gamma_{j}$ in $D^{n}$, and so to an orientation of the corresponding edge of $\Gamma$.

The second additional piece of structure is a labelling function $\lambda: E(\Gamma) \rightarrow V(\Gamma)$. Briefly, if $e$ is the edge corresponding to $\gamma_{j}$, then $\lambda(e)$ is defined to be the vertex corresponding to the component of $D^{n}-\left(\gamma_{1} \cup \cdots \cup \gamma_{m}\right)$ containing $\gamma_{j}^{\prime}$.

Thus we have obtained a labelled oriented tree $\Gamma$. It turns out that $\Gamma$ contains enough information to recover the homotopy type of the complement of the ribbon disc $k: D^{n} \hookrightarrow D^{n+2}$ associated to the ribbon immersion $i$. The dual graph of the ribbon immersion of Figure 1 is illustrated in Figure 4.

Note that $\Gamma$ does not determine the isotopy type of $k\left(D^{n}\right)$, or even of the ribbon knot $i\left(\partial D^{n}\right)=k\left(\partial D^{n}\right)$. For example, put $n=2$ and give one of the "ribbons" in Figure 1 a full twist. This alters the isotopy type of $i\left(\partial D^{2}\right)$ without altering $\Gamma$.

Abstract labelled oriented trees are discussed in $§ 3$.

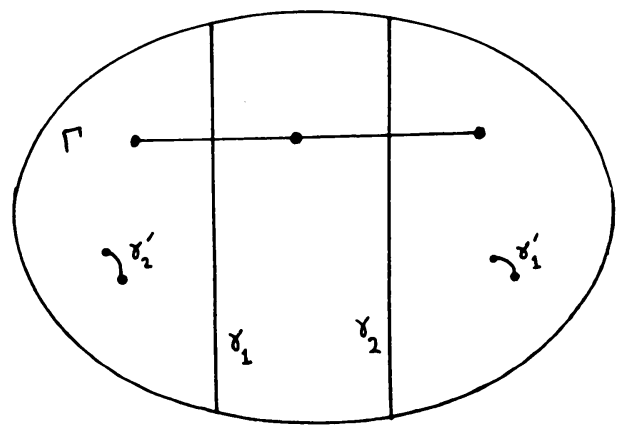

Figure 3 


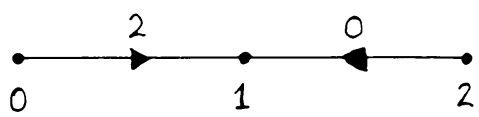

FIGURE 4

3. Labelled oriented trees. A labelled oriented graph $\Gamma$ consists of two finite sets $V=V(\Gamma)$ and $E=E(\Gamma)$, and three maps $\iota, \tau . \lambda: E \rightarrow V$. The set $V$ is the iertex set of $\Gamma$. and $E$ is the edge set of $\Gamma$. The maps $\iota$ and $\tau$ are the initial and terminal certex maps respectively, and define the underlying oriented graph structure of $\Gamma$. The map $\lambda$ is called the labelling function.

Since the labelling function $\lambda$ plays a different rôle from the maps $\iota$ and $\tau$. we can radically alter the labelled oriented graph $\Gamma$ by permuting the three maps $\iota . \tau$ and $\lambda$. This idea will be exploited in $\$ 10$.

We will be concerned mainly with labelled oriented trees. that is. labelled oriented graphs $(V, E, \iota, \tau, \lambda)$ whose underlying graph structures $(V, E, \iota, \tau)$ are trees. However. most of the constructions of this section work for general graphs. and some of the applications in $\$ 10$ also hold in the general case.

Given a labelled oriented graph $\Gamma$. we construct a presentation $P(\Gamma)$ of a group $G(\Gamma)$ as follows:

$$
P(\Gamma):\left\langle V(\Gamma) \mid\left\{\tau(e)^{-1} \lambda(e)^{-1} \iota(e) \lambda(e): e \in E(\Gamma)\right\}\right\rangle .
$$

We will of ten use integers to denote vertices of $\Gamma$. and then it is less confusing to use the elements of $V(\Gamma)$ as subscripts for the generators of $P(\Gamma)$. rather than to denote the generators themselves. We will then write $x_{u}, x_{r}$. etc.. instead of $u, v$. For example. if $\Gamma$ is the labelled oriented tree in Figure 4. then $P(\Gamma)$ is the presentation

$$
\left\langle x_{0}, x_{1}, x_{2} \mid x_{1}^{-1} x_{2}^{-1} x_{0} x_{2}, x_{1}^{-1} x_{0}^{-1} x_{2} x_{0}\right\rangle \text {. }
$$

There is a 2-complex $K(\Gamma)$ associated to $P(\Gamma)$ in the usual way. Namely. $K(\Gamma)$ has a single 0-cell, a 1-cell for each generator of $P(\Gamma)$ (in other words, for each vertex of $\Gamma$ ). and a 2-cell for each relator of $P(\Gamma)$ (in other words. for each edge of $\Gamma$ ). The 2 -cells are sewn on in such a way as to "spell" the relators. It follows from the special form of the relators of $P(\Gamma)$ that $K(\Gamma)$ has the same homology as the suspension $S\left(\Gamma^{*}\right)$, where " " means "add a disjoint base point". In particular. if $\Gamma$ is a tree, then $K(\Gamma)$ is a homology circle.

Proposition 3.1. For each integer $n \geqslant 2$, ever. labelled oriented tree is isomorphic to the dual graph of some ribbon immersion $D^{n} \rightarrow S^{n-1}$.

We omit the proof. It is merely a matter of following the construction of $\$ 2$ in reverse. The labelled oriented tree gives the pattern for the self-intersections of the ribbon immersion. and the condition $n \geqslant 2$ ensures that there is enough elbow-room in $S^{n-1}$ to realise this pattern.

Proposition 3.2. Let $i: D^{n} \hookrightarrow S^{n-1}$ be a ribbon immersion. let $k: D^{n} \hookrightarrow D^{n-2}$ be the corresponding ribbon disc. and let $\Gamma$ be the dual graph of $i$. Then $K(\Gamma)$ embeds in $D^{\prime \prime-2}-k\left(D^{n}\right)$ as a spine. 
This is more or less standard. One can arrange $k$ so that the Morse function $r \circ k$ : $D^{n} \rightarrow \mathbf{R}$ gives rise to a handle decomposition of $D^{n+2}-k\left(D^{n}\right)$ which is modelled on the 2-complex $K(\Gamma)$. It is then routine to embed $K(\Gamma)$ as a spine in $D^{n+2}-k\left(D^{n}\right)$ using the handle cores (see $[3,8,9]$ for more details).

Since we are interested only in the homotopy groups of $D^{n+2}-k\left(D^{n}\right)$, it makes sense to consider transformations of a labelled oriented tree $\Gamma$ which do not affect the homotopy type of $K(\Gamma)$. Below are three examples of such moves.

M1. Suppose $\lambda(e)=\iota(e)$ or $\lambda(e)=\tau(e)$ for some edge $e$ of $\Gamma$. Form a tree $\Gamma^{\prime}$ from $\Gamma$ by shrinking $e$ to a point. Then $E\left(\Gamma^{\prime}\right)$ may be regarded as $E(\Gamma)-\{e\}$ and there is an identification map $q: V(\Gamma) \rightarrow V\left(\Gamma^{\prime}\right)$. The labelling function $\lambda^{\prime}$ for $\Gamma^{\prime}$ is given by $\lambda^{\prime}=(q \circ \lambda) \mid E\left(\Gamma^{\prime}\right)$.

M2. Suppose, for two distinct edges $e$ and $f$ of $\Gamma$, that $\lambda(e)=\lambda(f)$ and either $\iota(e)=\iota(f)$ or $\tau(e)=\tau(f)$. Form $\Gamma^{\prime}$ from $\Gamma$ by a "fold" which identifies $e$ with $f$. Then $\lambda$ induces a well-defined labelling function $\lambda^{\prime}$ for $\Gamma^{\prime}$, since $\lambda(e)=\lambda(f)$.

M3. Suppose $v$ is an extremal vertex of $\Gamma$ which does not appear as a label $\lambda(e)$ for any edge $e$. Omit $v$ and the edge incident at $v$ from $\Gamma$.

We will call a labelled oriented tree $\Gamma$ reduced if none of these moves can be applied to it. Clearly any labelled oriented tree can be changed to a reduced one by finitely many moves.

4. Weakly labelled oriented trees. The dual graph of a ribbon immersion corresponds to a decomposition of $D^{n}$ into $m$ ribbons $N_{1}, \ldots, N_{m}$ (regular neighbourhoods of the separating discs $\gamma_{j}$ ), and $m+1$ subdiscs (the closures of the connected components of $\left.D^{n}-\left(N_{1} \cup \cdots \cup N_{m}\right)\right)$, such that each ribbon contains precisely one separating disc and no nonseparating discs. Such ribbon decompositions are more specialised than are usually considered (see, for example, [3,9]). Normally arbitrary decompositions are allowed, provided that the separating discs all lie in ribbons and the nonseparating discs lie in subdiscs. Thus for example the immersion in Figure 1 is usually regarded as two subdiscs connected by a single ribbon, which intersects each subdisc once. Corresponding to this more general type of decomposition, one has a more general type of labelled oriented tree.

Definition. A weakly labelled oriented tree consists of an oriented tree $\Gamma=$ $(V, E, \iota, \tau)$, together with a weak labelling function $\lambda: E \rightarrow F$, where $F$ is the free group with basis $V$.

As with the generators of the presentation $P(\Gamma)$ in $\S 3$, it is sometimes convenient to write the basis elements of $F$ as $x_{u}, x_{v}$, etc. instead of $u, v$. Exactly as in $\S 3$, we associate to every weakly labelled oriented tree $\Gamma$ a presentation

$$
P(\Gamma):\left\langle V(\Gamma) \mid\left\{\tau(e)^{-1} \lambda(e)^{-1} \iota(e) \lambda(e) ; e \in E(\Gamma)\right\}\right\rangle
$$

of a group $G(\Gamma)$, and hence a 2-complex $K(\Gamma)$, which is a homology circle with $\pi_{1} K(\Gamma)=G(\Gamma)$.

Clearly these generalise the definitions in $\S 3$, if we identify $V(\Gamma)$ with a subset of $F$, and so regard labelled oriented trees as special cases of weakly labelled oriented trees. It is possible, as in $\S 3$, to consider also the case where $\Gamma$ is not a tree but an 
arbitrary graph. We will not do this here, however, as the more general case will not arise in our later applications. There are also analogues of Propositions 3.1 and 3.2 for weakly labelled oriented trees, whose formulation we leave to the reader.

As in $\S 3$, we consider transformations of $\Gamma$ which do not affect the homotopy type of $K(\Gamma)$. Indeed, the main advantage of weakly labelled oriented trees is that more subtle moves are possible. The moves we allow are the following.

M1. As in $\S 3$.

M2. As in $\S 3$.

M3. Suppose $v$ is an extremal vertex of $\Gamma$ such that no label $\lambda(e)$ involves the letter $v$, then omit $v$ and the edge incident at $v$ from $\Gamma$.

M4. Switch the orientation of some edge $e$ of $\Gamma$, and replace $\lambda(e)$ by $\lambda(e)^{-1}$.

M5. Suppose $\tau(e)=\iota(f)$. Then "slide $f$ along $e$ ", in other words change $\iota(f)$ to $\iota(e)$ and replace $\lambda(f)$ by $\lambda(e) \lambda(f)$.

M6. Replace an occurrence of the letter $\tau(f)$ in the word $\lambda(e)$ by $\lambda(f)^{-1} \iota(f) \lambda(f)$, where $e$ and $f$ are distinct edges of $\Gamma$.

We say that two weakly labelled oriented trees $\Gamma_{1}$ and $\Gamma_{2}$ are equivalent if $\Gamma_{2}$ can be obtained from $\Gamma_{1}$ by a finite sequence of moves of types M1-M6, or the inverses of these moves. If $\Gamma_{1}$ and $\Gamma_{2}$ have a common subgraph $\Delta$ which is left unaltered by these moves, then we say that $\Gamma_{1}$ and $\Gamma_{2}$ are equivalent rel $\Delta$.

PROPOSITION 4.1. Every equivalence class of weakly labelled oriented trees contains:

(i) a star, that is a tree of diameter at most 2;

(ii) a chain, that is a tree with only two extremal vertices;

(iii) a labelled oriented tree;

(iv) a reduced labelled oriented tree;

(v) a labelled oriented chain.

REMARKS. The constructions of ribbon discs in [3 and 9] are essentially modelled on weakly labelled oriented trees which are, respectively, stars and chains. The Wirtinger presentation of any classical knot group becomes, after discarding a redundant relator, equal to $P(\Gamma)$ for some reduced labelled oriented chain $\Gamma$. The converse is false: if $\Gamma$ is the reduced labelled oriented chain in Figure 4, then $G(\Gamma)$ has infinitely divisible elements, and so cannot be a 3-manifold group [16].

The effect on $P(\Gamma)$ of applying one of the moves M1-M6 to $\Gamma$ can be achieved by some combination of the following moves on presentations [17]:

Expansion. Introduce a new generator $a$ and a relator $a w$, where $w$ is a word in the existing generators.

Contraction. The reverse of expansion.

Replacement. Replace a relator $r$ by $r w$, where $w$ is a consequence of the remaining relators.

These are special cases of the extended Nielsen transformations of Andrews and Curtis [2]. In particular they translate to formal deformations of the corresponding 2-complexes, involving only cells in dimensions up to 3 . We will say that two presentations are equivalent (rel a subpresentation) if one can be obtained from the other by a sequence of expansions, contractions and replacements (leaving the subpresentation fixed). The above discussion can be summarised as follows. 
Proposition 4.2. Suppose $\Gamma_{1}$ and $\Gamma_{2}$ are weakly labelled oriented trees with a common subgraph $\Delta$ such that $\Gamma_{1}$ and $\Gamma_{2}$ are equivalent rel $\Delta$. Then $P\left(\Gamma_{1}\right)$ and $P\left(\Gamma_{2}\right)$ are equivalent rel $P(\Delta)$, and so $K\left(\Gamma_{1}\right)$ is 3-deformable to $K\left(\Gamma_{2}\right)$ rel $K(\Delta)$.

5. Indicability and reducibility. In this section we recall some definitions and results from [11] concerning locally indicable groups and reducible presentations and 2-complexes.

Definition. A group is locally indicable if each of its nontrivial finitely generated subgroups admits an infinite cyclic homomorphic image.

FACT 5.1. If $X$ is a connected 2-complex, with $\pi_{1} X$ locally indicable and $H_{2}(X ; \mathbf{Z})=0$, then $X$ is aspherical.

This fact applies in particular to the case where $X=K(\Gamma)$ for some (weakly) labelled oriented tree $\Gamma$, for then $X$ is a homology circle. Thus, in order to show that $X$ is aspherical, it is sufficient to show that $G(\Gamma) \cong \pi_{1} X$ is locally indicable. In particular, Theorem A follows from Theorem B.

For a direct proof of Fact 5.1, see [11, Theorem 5.2]. Alternatively, use the fact $[7,13]$ that locally indicable groups are Z-conservative. In other words, whenever $\tilde{X} \rightarrow X$ is a regular covering of 2-complexes, with locally indicable covering transformation group, and $H_{2}(X)=0$, then $H_{2}(\tilde{X})=0$. Now apply this to the universal covering of $X$ to get $\pi_{2} X=0$.

In order to be able to apply Fact 5.1, it is useful to have some criteria for local indicability. Recall [11] that an elementary reduction is a pair $(X, Y)$ of 2-complexes, with $Y \subset X$, such that $X-Y$ consists of precisely one 1-cell $e^{1}$, together with at most one 2-cell. Furthermore the 2-cell, if it exists, is required to properly involve $e^{1}$, in the sense that its attaching map is not homotopic (in $Y \cup e^{1}$ ) to a map into $Y$.

A 2-complex is reducible if, for every finite subcomplex $X$ of dimension at least 1 , there exists an elementary reduction $(X, Y)$. A pair $(L, K)$ of 2-complexes, with $K \subset L$, is reducible if, for every finite subcomplex $X$ of $L$, either $X \subset K \cup L^{(0)}$ (where $L^{(i)}$ denotes the $i$-skeleton of $L$ ), or there exists an elementary reduction $(X, Y)$ with $K \cap X \subset Y$.

A presentation (resp. pair of presentations) is reducible if the corresponding 2-complex (resp. pair of 2-complexes) is reducible. We will also say that a 2-complex $L$ (resp. presentation $Q$ ) reduces to the subcomplex $K$ (resp. subpresentation $P$ ) if the pair $(L, K)$ (resp. $(Q, P))$ is reducible.

A relator $r$ in a presentation is a proper power if it is equivalent, modulo the remaining relators, to a word $r^{\prime}=s^{m}$ for some word $s$ and some integer $m \geqslant 2$.

FACT 5.2. If $G$ has a reducible presentation in which no relator is a proper power, then $G$ is locally indicable.

FACT 5.3. If $(Q, P)$ is a reducible pair of presentations, no relator of $Q-P$ is a proper power in $Q$, and $P$ presents a locally indicable group, then $Q$ presents a locally indicable group.

Fact 5.2 is [11, Corollary 4.5]. In particular, torsion free one-relator groups are locally indicable (see also [4]). Fact 5.3 can be proved using the same arguments as Fact 5.2, using [11, Theorem 4.2] as an inductive step. 
Now a relator which is a proper power in a presentation $P$ determines a nonzero element of $\mathrm{H}_{2}\left(X, \mathbf{Z}_{p}\right)$ for some prime $p$, where $X$ is the 2-complex model of $P$. The 2-complexes $X$ to be considered in this paper are all of special forms, namely either:

(i) homology circles, such as $K(\Gamma)$ for weakly labelled oriented trees $\Gamma$;

(ii) infinite cyclic covers of homology circles; or

(iii) (2-complexes homotopy equivalent to) subcomplexes of infinite cyclic covers of 2-dimensional homology circles.

That $H_{2}\left(X ; \mathbf{Z}_{p}\right)=0$ is obvious in (i). In (ii) it follows from [1, Proposition 1], and in (iii) it follows from (ii). Hence the "no proper power" condition in 5.2 and 5.3 will always be automatically satisfied for any presentation or 2-complex which occurs in the remainder of the paper. From now on we will make no explicit mention of this condition, but concentrate on the question of reducibility when we want to apply 5.2 or 5.3 .

6. Sloping words. Let $F$ be the free group on a set $A$. We define sloping words in $A$ as follows. The empty word is the unique sloping word of height 0 . Inductively, for $k \geqslant 1$, a sloping word of height $k$ is any word of the form

$$
a^{w}=w^{-1} a w,
$$

where $a \in A \cup A^{-1}$ and $w$ is a sloping word of height $k-1$.

To each sloping word of height greater than 0 , we associate an element of $A$ called its uppermost letter as follows. If $a \in A$, then $a$ is the uppermost letter of each of the sloping words $a$ and $a^{-1}$. If $w_{1}=a^{n}$ is a sloping word of height $k \geqslant 2$, where $a \in A \cup A^{-1}$ and $w$ is a sloping word of height $k-1$, then $w_{1}$ has the same uppermost letter as $w$.

Intuitively, a sloping word is one which can be written, for example

$$
a^{b^{c}} \quad\left(=c^{-1} b^{-1} c a c^{-1} b c\right) \quad\left(a, b, c \in A \cup A^{-1}\right)
$$

in the case of height 3 , and the uppermost letter is the symbol appearing at the top right-hand side (in this case $c$ ) or its inverse. More complicated sloping words rapidly become difficult to print in this form. Regarded as an ordinary word in $A$, a sloping word of height $k$ has length $2^{k}-1$, and every alternate symbol is the uppermost letter or its inverse. Sloping words are not in general reduced words. By abuse of notation we will refer to an element of $F$ as reduced sloping word if its reduced form is a sloping word.

Recall that the total exponent of a word $w=a_{1}^{e(1)} \cdots a_{n}^{e(n)}\left(a_{i} \in A, e(i)=\mp 1\right)$ is $e(1)+\cdots+e(n)$. Clearly sloping words have total exponent $\mp 1$. An initial segment of $w$ is a word $a_{1}^{e(1)} \cdots a_{m}^{e(m)}$ for some $m$ with $0 \leqslant m \leqslant n$. Thus a sloping word of height $k$ has $2^{k}$ initial segments.

LEMma 6.1. Let $w$ be a sloping word of height $k$. Then the sequence of total exponents of initial segments of $w$ achieves each of its maximum and minimum values precisely once, and these two extremes differ by $k$.

Proof. The proof is by induction on the height $k$. If $k=0$, then the sequence in question consists of the single value 0 , and the result is trivial. 
Suppose then that $w=x^{-1} a x$, where $a \in A \cup A^{-1}$ and $x$ is a sloping word of height $k-1$, and that the conclusion of the lemma holds for $x$. That is, the sequence

$$
0=m_{1}, \ldots, m_{s}=\mp 1 \quad\left(s=2^{k-1}\right)
$$

of total exponents of initial segments of $x$ achieves its maximum and minimum values once each, say at the $p$ th and $q$ th places respectively, and $m_{p}-m_{q}=k-1$.

Suppose first that $a \in A$. Then the sequence of exponent sums of initial segments of $w$ is

$$
0, m_{s-1}-m_{s}, \ldots,-m_{s}, 1-m_{s}, \ldots, 1+m_{s-1}-m_{s}, 1 .
$$

This sequence achieves its maximum value precisely once, at the $\left(2^{k+1}+p\right)$ th term $1+m_{p}-m_{s}$, and its minimum value precisely once, at the $\left(2^{k-1}-q\right)$ th term $m_{q}-m_{s} ;$ and moreover

$$
\left(1+m_{p}-m_{s}\right)-\left(m_{q}-m_{s}\right)=k \text {. }
$$

This completes the inductive step when $a \in A$. A similar argument works when $a \in A^{-1}$.

Our main interest in sloping words is the following

THEOREM 6.2. Let $\Gamma$ be a weakly labelled oriented tree with 3 vertices, in which at least one label is a reduced sloping word. Then $G(\Gamma)$ is locally indicable.

Proof. We have $P(\Gamma)=\left\langle x_{0}, x_{1}, x_{2} \mid x_{0}^{-1} w_{1}^{-1} x_{1} w_{1}, x_{1}^{-1} w_{2}^{-1} x_{2} w_{2}\right\rangle$, up to cyclic rearrangement of the relators, where $w_{1}$ is a reduced sloping word of height $k \geqslant 0$, and $w_{2}$ is an arbitrary word. If $k \leqslant 1$, then $P(\Gamma)$ is equivalent to a 1-relator presentation, and the result follows from Fact 5.2. Indeed this equivalence is induced by an equivalence of weakly labelled oriented trees. (If $w_{1}=1$ or $x_{0}^{\mp 1}$ or $x_{1}^{\mp 1}$ this is obvious. If $w_{1}=x_{2}^{\mp 1}$ use M6 moves to remove all occurrences of $x_{0}$ from $w_{2}$, and then an M3 move to elmiminate $x_{1}$ and the first relator.)

Suppose then that $k \geqslant 2$. Let $X=K(\Gamma)$ and let $\tilde{X}$ be the maximal abelian cover of $X$. To avoid proliferation of subscripts, replace the generators $x_{0}, x_{1}, x_{2}$ by $a, b, c$ respectively. Write $R, S$ for the 2-cells of $X$ corresponding to the relators $a^{-1} w_{1}^{-1} b w_{1}$, $b^{-1} w_{2}^{-1} c w_{2}$ respectively, and assume that $R$ and $S$ are attached along cyclically reduced closed paths in $X^{(1)}$.

Since the covering transformation group of $\tilde{X} \rightarrow X$ is infinite cyclic, we may index the 0 -cells of $X$ by integers. Since $a, b, c$ represent the same generator of this cyclic group, every 1-cell of $X$ connects two 0 -cells with adjacent indices. For each integer $i$, let $a_{i}, b_{i}, c_{i}$ denote the 1-cells which cover $a, b, c$, respectively, and which connect the 0 -cells $i$ and $i+1$. These 1-cells are naturally oriented from $i$ to $i+1$.

Next consider a 2-cell $R^{\prime}$ of $X$ which covers $R$. The sequence of 0 -cells traversed by an attaching path for $R^{\prime}$ is, up to reversal and cyclic permutation,

$$
C, C+m_{1}, \ldots, C+m_{t}=C
$$

for some constant $C$, where $0, m_{1}, \ldots, m_{t}$ is the sequence of total exponents of initial segments of some cyclically reduced conjugate of $a^{-1} w_{1}^{-1} b w_{1}$. If the uppermost letter of $w_{1}$ is $c$, then $a^{-1} w_{1}^{-1} b w_{1}$ is cyclically reduced as written, and it follows from 
Lemma 6.1 that this sequence of 0 -cells achieves its maximum and its minimum value once each, and that these values differ by $k+1$. If the uppermost letter of $w_{1}$ is either $a$ or $b$, then precisely one pair of letters cancel in the process of cyclically reducing $a^{-1} w_{1}^{-1} b w_{1}$ (since $w_{1}$ is reduced). The effect of this cancellation on the sequence of total exponents of initial segments is to remove two consecutive terms. Since these terms have consecutive values, and since $k \geqslant 2$, the removal of these terms may affect the maximum value of the sequence, or the minimum value, but not both. We will assume that the minimum value is not affected. The proof in the other case is similar. Hence we are assuming that the sequence of 0 -cells traversed by an attaching path for $R^{\prime}$ attains its minimum value precisely once. Clearly the same property must hold for any translate of $R^{\prime}$ by a covering transformation. For each integer $i$, let $R_{i}$ be that translate of $R^{\prime}$ for which the minimum 0 -cell is $i$.

Similarly, let $S_{i}$ denote the 2-cell of $X$ which covers $S$, and for which the sequence of 0 -cells traversed by an attaching path takes the minimum value $i$.

Let $K$ be a finite connected subcomplex of $\tilde{X}$. We will find a connected subcomplex $L$ of $\tilde{X}$ with $K \subset L$ and $\pi_{1} L$ locally indicable. Since $\pi_{1} \tilde{X}$ is the direct limit of the fundamental groups of its finite connected subcomplexes, this is sufficient to prove that $\pi_{1} \tilde{X}$, and hence also $\pi_{1} X \cong G(\Gamma)$, is locally indicable.

Let $d \in\{a, b, c\}$ be such that $d_{0}$ occurs in (the attaching path for) $R_{0}$. Then $d_{0}$ is a free edge for $R_{0}$, that is $R_{0}$ has an attaching path $d_{0} p_{0}^{-1}$ for some path $p_{0}$ which does not involve $d_{0}$. Let $S_{0}^{(1)}$ denote the path obtained from (the attaching path for) $S_{0}$ by replacing each occurrence of $d_{0}$ by $p_{0}$ and then cyclically reducing. Call $S_{0}^{(1)}$ the first rewrite of $S_{0}$. Similarly, $d_{1}$ is a free edge of $R_{1}, R_{1}=d_{1} p_{1}^{-1}$, and we can form the first rewrite $S_{1}^{(1)}$ of $S_{1}$ and the second rewrite $S_{0}^{(2)}$ of $S_{0}$ by replacing each occurrence of $d_{1}$ in $S_{1}, S_{0}^{(1)}$ respectively by $p_{1}$ and then cyclically reducing. Continuing in this way, we define the ith rewrite $S_{j}^{(i)}$ of $S_{j}$ for all $i \geqslant 1$ and all $j \geqslant 0$.

Define $\rho, \sigma$ to be the maximum 0-cells traversed by the attaching paths for $R_{0}, S_{0}$ respectively, and define $\alpha_{i}$ to be the minimum 0 -cell traversed by $S_{0}^{(i)}$ for all $i \geqslant 1$. Then the increasing sequence of integers $\left\{\alpha_{i}\right\}$ is bounded above by $\rho+\sigma$, and so is eventually constant. To see this, suppose $\alpha=\alpha_{n} \geqslant \rho+\sigma+1$ for some $n$. Let $Y$ be the greatest subcomplex of $X$ containing only those 0 -cells $j \in \mathbf{Z}$ with $0 \leqslant j \leqslant(n+$ $\rho+\sigma)$. The 1-cells of $Y$ are $a_{j}, b_{j}, c_{j}(0 \leqslant j \leqslant(n+\rho+\sigma-1))$, and the 2-cells are $R_{j}(0 \leqslant j \leqslant(n+\sigma))$ and $S_{j}(0 \leqslant j \leqslant(n+\rho))$.

Let $Y^{\prime}$ be the 2-complex obtained from $Y$ by replacing $S_{j}$ by a 2 -cell attached along the $n$th rewrite $S_{j}^{(n)}$ for $0 \leqslant j \leqslant(\rho+\sigma)$. Then $Y^{\prime}$ is homotopy equivalent to $Y$, and furthermore $Y^{\prime}$ collapses onto the subcomplex $Y^{\prime \prime}$ obtained by removing $R_{0}, \ldots, R_{\rho+\sigma}$ and $d_{0}, \ldots, d_{\rho+\sigma}$ from $Y^{\prime}$. Let $Z$ denote the 1-dimensional subcomplex of $Y^{\prime \prime}$ consisting of the 0 -cells $0, \ldots, \rho+\sigma+1$ and the 1-cells $a_{j}, b_{j}, c_{j}$ for $0 \leqslant j \leqslant \rho+\sigma$. Since the minimum 0 -cell in $S_{j}^{(n)}$ is $j+\alpha \geqslant j+\rho+\sigma+1$ for all $j$, the identity map on $Z$ extends to a map $Y^{\prime \prime} \rightarrow Z$ which sends all of $Y^{\prime \prime}-Z$ to the 0 -cell $\rho+\sigma+1$. Hence

$$
\beta_{1}(Y)=\beta_{1}\left(Y^{\prime \prime}\right) \geqslant \beta_{1}(Z)=\rho+\sigma+1
$$

Now $H_{2}\left(Y ; \mathbf{Z}_{p}\right)=0$ for any prime $p$ (see $\left.\S 5\right)$, so $\beta_{2}(Y)=0$. Also $\beta_{0}(Y)=1$ since $Y$ is connected. Hence

$$
\chi(Y) \leqslant-\rho-\sigma .
$$


On the other hand, a direct computation gives

$$
\chi(Y)=1-\rho-\sigma,
$$

since $Y$ consists of $(n+\rho+\sigma+1) 0$-cells, $3(n+\rho+\sigma) 1$-cells, $(n+\sigma) 2$-cells $R_{j}$, and $(n+\rho) 2$-cells $S_{j}$. Hence we have a contradiction, so $\alpha_{n} \leqslant \rho+\sigma$ for all $n$, as claimed.

Now let us return to our finite subcomplex $K \subset \tilde{X}$. By applying a covering transformation if necessary, we may assume that 0 is the minimum 0 -cell in $K$. Let $m$ be the maximum 0 -cell in $K$, and choose $n$ large enough so that $n \geqslant \rho+\sigma$ and $\alpha_{n+i}=\alpha_{n}=\alpha$ for all $i \geqslant 0$. Take $L$ to be the subcomplex of $X$ consisting of all 0 -cells $j$ with $0 \leqslant j \leqslant m+n+\rho-\sigma$; all 1 -cells $a_{j}, b_{j}, c_{j}$ with $0 \leqslant j \leqslant m+n+\rho-$ $\sigma-1$; all 2-cells $R_{j}$ with $0 \leqslant j \leqslant m+n-\sigma$; and all 2-cells $S_{j}$ with $0 \leqslant j \leqslant m-\sigma$.

Now replace each 2-cell $S_{j}$ by its $(m+n-\sigma-j)$ th rewrite to get a 2-complex $Y^{\prime}$ homotopy equivalent to $Y$. Then $Y^{\prime}$ collapses to the subcomplex $L^{\prime \prime}$ obtained by removing the 2-cells $R_{j}$ and the 1-cells $d_{j}$ for $0 \leqslant j \leqslant m+n-\sigma$. The 2-cells of $L^{\prime \prime}$ are attached by cyclically reduced closed paths $S_{j}^{(m+n-\sigma-j)}(0 \leqslant j \leqslant m-\sigma)$, and the minimum 0 -cell traversed by $S_{j}^{(m+n-\sigma-j)}$ is $j+\alpha$, so it readily follows that $L^{\prime \prime}$ is reducible. Hence $\pi_{1} L \cong \pi_{1} L^{\prime \prime}$ is locally indicable, by Fact 5.2.

Clearly $K \subset L$, so the proof of Theorem 6.2 is complete.

7. Admissible subgraphs and generating sets. Let $\Gamma$ be a (weakly) labelled oriented tree, and $\Gamma^{\prime}$ a subgraph of $\Gamma$. We call $\Gamma^{\prime}$ an admissible subgraph if it is closed with respect to the labelling function $\lambda$, that is if $\lambda(e)$ is in $V\left(\Gamma^{\prime}\right)$ (resp. a word in $V\left(\Gamma^{\prime}\right)$ ) for any edge $e$ of $\Gamma^{\prime}$. There are several fairly trivial examples of admissible subgraphs of any given (weakly) labelled oriented tree $\Gamma$. For example $\Gamma$ itself is admissible, as is any subgraph with no edges. The interesting cases, however, are when $\Gamma$ has a nontrivial admissible subtree (see for example Corollary 7.2).

Let $V_{0} \subset V(\Gamma)$ be a subset of the vertex set of the weakly labelled oriented tree $\Gamma$. We say that the subgraph $\Gamma^{\prime}$ of $\Gamma$ is generated by $V_{0}$ if:

(1) $\Gamma^{\prime}$ is admissible;

(2) $V_{0} \subset V\left(\Gamma^{\prime}\right)$;

(3) no edge of $\Gamma-\Gamma^{\prime}$, incident at a vertex of $\Gamma^{\prime}$, is labelled by a word in $V\left(\Gamma^{\prime}\right)$;

(4) $\Gamma^{\prime}$ is minimial with respect to (1)-(3). Clearly any subset $V_{0} \subset V(\Gamma)$ generates a unique subgraph $\Gamma^{\prime}$.

THEOREM 7.1. Let $\Gamma^{\prime}$ be an admissible subgraph of the weakly labelled oriented tree $\Gamma$, such that $\Gamma$ is generated by $V\left(\Gamma^{\prime}\right)$. Let $\Delta$ be the smallest subtree of (the underlying graph of ) $\Gamma$ which contains $\Gamma^{\prime}$. Then there exist an orientation and a weak labelling for $\Delta$ such that:

(i) the orientations and weak labelling of $\Gamma$ and $\Delta$ agree on $\Gamma^{\prime}$;

(ii) $\Delta$ is equivalent to $\Gamma$ rel $\Gamma^{\prime}$;

(iii) the labels on edges of $\Delta$ are words in $V\left(\Gamma^{\prime}\right)$.

Suppose in addition that each vertex of $\Gamma-\Gamma^{\prime}$ is extremal in $\Gamma$, and that each edge of $\Gamma-\Gamma^{\prime}$ is labelled either by a vertex in $V(\Gamma)-V\left(\Gamma^{\prime}\right)$, or by a reduced sloping word of height at least 1 in $V\left(\Gamma^{\prime}\right)$, whose uppermost letter is an extremal vertex of $\Gamma$. Then $V(\Delta)=V\left(\Gamma^{\prime}\right)$, and the edges of $\Delta-\Gamma^{\prime}$ can also be labelled by reduced sloping words. 
Proof. The proof is by induction on the number of edges in $\Gamma-\Gamma^{\prime}$. In the initial case $\Gamma=\Delta=\Gamma^{\prime}$ and the result is trivial.

Now suppose $\Gamma \neq \Gamma^{\prime}$. Then there exists an edge $e$ of $\Gamma-\Gamma^{\prime}$, incident at a vertex $u$ of $\Gamma^{\prime}$, and labelled by a word in $V\left(\Gamma^{\prime}\right)$. For otherwise $\Gamma^{\prime}$ satisfies conditions (1)-(3) in the definition of the subgraph generated by $V\left(\Gamma^{\prime}\right)$, which by hypothesis is $\Gamma$. By condition (4) we have $\Gamma \subset \Gamma^{\prime}$, a contradiction. By applying a move of type M4 if necessary, we may assume $u=\iota(e)$. Let $v=\tau(e)$. We distinguish 3 cases.

Case 1. $v \in V\left(\Gamma^{\prime}\right)$. Then $e \in E(\Delta)$. Let $\Gamma^{\prime \prime}$ be the subgraph consisting of $\Gamma^{\prime}$ together with $e$. Then $\Gamma^{\prime \prime}$ is admissible and $V\left(\Gamma^{\prime \prime}\right)=V\left(\Gamma^{\prime}\right)$. Replace $\Gamma^{\prime}$ by $\Gamma^{\prime \prime}$ and apply the inductive hypothesis.

Case 2. $v \in V(\Delta)-V\left(\Gamma^{\prime}\right)$. Let $\Gamma^{\prime \prime}$ be the subgraph consisting of $\Gamma^{\prime}$ together with $e$ and $v$. Then $\Gamma^{\prime \prime}$ is admissible and $V\left(\Gamma^{\prime \prime}\right)=V\left(\Gamma^{\prime}\right) \cup\{v\}$. Replace $\Gamma^{\prime}$ by $\Gamma^{\prime \prime}$ and apply the inductive hypothesis, as in Case 1. The result follows, provided we can alter the labels in $\Delta$ so that they do not involve $v$. But by hypothesis $\lambda(e)$ is a word in $V\left(\Gamma^{\prime}\right)$, so does not involve $v$. Hence we may remove all occurrences of $v$ in labels of $\Delta$ by a series of moves of type M6, replacing any such occurrence by $\lambda(e)^{-1} u \lambda(e)$.

Case 3. $v \in V(\Gamma)-V(\Delta)$. If $f$ is any edge of $\Gamma$ incident at $v$, and distinct from $e$, we may first apply a move M4 to $f$ if necessary to ensure that $\iota(f)=v$, and then a move M5 to slide $f$ along $e$. This reduces the index of $v$ without affecting $\Delta$. Thus, after a series of such moves, we can assume that $v$ is an extremal vertex of $\Gamma$, with $e$ the only edge incident at $v$. Since $v$ does not occur in $\lambda(e)$, we may apply a series of moves M6 to replace any occurrence of $v$ in a label of $\Gamma$ by $\lambda(e)^{-1} u \lambda(e)$, and remove all occurrences of $v$ from labels of $\Gamma$. Finally, we can apply a move M3 to remove $e$ and $v$ from $\Gamma$.

Let $\Gamma_{1}$ be the tree obtained from $\Gamma$ after the sequence of moves above. Then the hypotheses of the first part of the theorem apply to $\Gamma_{1}$ and $\Gamma^{\prime}$. Apply the inductive hypothesis to obtain the result, since $\Gamma$ is clearly equivalent to $\Gamma_{1}$ rel $\Gamma^{\prime}$.

This completes the proof of the first part of the theorem. Suppose now that the additional hypotheses of the second part also hold. Then it is immediate that $V(\Delta)=V\left(\Gamma^{\prime}\right)$. In particular it follows that Case 2 in the inductive step cannot occur. In Case 1 no labels are altered, so we need only consider Case 3, in which case it is sufficient to show that the additional hypotheses also hold for the tree $\Gamma_{1}$.

Note first that no sliding moves M5 are necessary, since $v$ is already extremal in $\Gamma$. Hence $\Gamma_{1}$ is obtained, as a graph, by omitting $v$ and $e$ from $\Gamma$. Clearly every vertex of $\Gamma_{1}-\Gamma^{\prime}$ is extremal in $\Gamma_{1}$.

Consider now an edge $f \neq e$ of $\Gamma-\Gamma^{\prime}$. The label $\lambda(f)$ of $f$ in $\Gamma$ is either a vertex of $\Gamma-\Gamma^{\prime}$ or a reduced sloping word in $V\left(\Gamma^{\prime}\right)$, by hypothesis. This label is altered in passing from $\Gamma$ to $\Gamma_{1}$ only if it involves $v$, that is only if $\lambda(f)=v$. But then the label of $f$ in $\Gamma_{1}$ is the sloping word $\lambda(e)^{-1} u \lambda(e)$. Now $\lambda(e)$ is a word in $V\left(\Gamma^{\prime}\right)$, by assumption. It is therefore a reduced sloping word, whose uppermost letter, $v^{\prime}$ say, is an extremal vertex in $\Gamma$. In particular, $v^{\prime} \neq u$, since $u$ is not extremal in $\Gamma$ (since $\Gamma$ has at least two edges $e, f$, we cannot have both $u=\iota(e)$ and $v=\tau(e)$ extremal in $\Gamma)$. Hence the sloping word $\lambda(e)^{-1} u \lambda(e)$ is reduced, with uppermost letter $v^{\prime}$, and $v^{\prime}$ is an extremal vertex in $\Gamma_{1}$. This completes the proof. 
COROllary 7.2. Let $\Gamma^{\prime}$ be an admissible subtree of the labelled oriented tree $\Gamma$, such that each vertex of $\Gamma-\Gamma^{\prime}$ is extremal in $\Gamma$. Then $P(\Gamma)$ is equivalent rel $P\left(\Gamma^{\prime}\right)$ to a presentation which reduces to $P\left(\Gamma^{\prime}\right)$.

Proof. By an easy inductive argument it suffices to consider the case where $\Gamma^{\prime}$ is a maximal proper admissible subtree.

Suppose first that some vertex $u$ of $\Gamma-\Gamma^{\prime}$ does not occur as a label in $\Gamma$. Then we may apply move $\mathrm{M} 3$ to remove $u$ and its incident edge, and obtain a proper subtree $\Gamma_{1} \subset \Gamma$ which is equivalent rel $\Gamma^{\prime}$ to $\Gamma$. Clearly $\Gamma_{1}$ is admissible, so $\Gamma_{1}=\Gamma^{\prime}$ by maximality of $\Gamma^{\prime}$, and $P(\Gamma)$ is equivalent to $P\left(\Gamma^{\prime}\right)$ rel $\Gamma P\left(\Gamma^{\prime}\right)$ by Proposition 4.2.

Next suppose that every vertex in $\Gamma-\Gamma^{\prime}$ occurs as a label in $\Gamma$-necessarily of an edge in $\Gamma-\Gamma^{\prime}$, since $\Gamma^{\prime}$ is admissible. Let $u$ be any vertex in $\Gamma-\Gamma^{\prime}$, and let $\Gamma^{\prime \prime}$ be the subgraph of $\Gamma$ generated by $V_{0}=V\left(\Gamma^{\prime}\right) \cup\{u\}$. Then $\Gamma^{\prime \prime}$ is a tree, for otherwise $\Gamma^{\prime \prime}-\Gamma^{\prime}$ contains more vertices than edges, so some vertex $v$ of $\Gamma^{\prime \prime}-\Gamma^{\prime}$ does not occur as a label in $\Gamma^{\prime \prime}$. But $v=\lambda(e)$ for some edge $e$ of $\Gamma$, by assumption, and $e$ is necessarily incident at some vertex of $\Gamma^{\prime \prime}$. This contradicts condition (3) in the definition of the subgraph generated by $V_{0}$. Hence $\Gamma^{\prime \prime}$ is an admissible subtree of $\Gamma$ which properly contains $\Gamma^{\prime}$, so $\Gamma^{\prime \prime}=\Gamma$ by the maximality of $\Gamma^{\prime}$.

Now apply the theorem to $\Gamma$ and the admissible subgraph $\Gamma^{\prime} \cup\{u\}$. Then $\Gamma$ is equivalent rel $\Gamma^{\prime}$ to a weakly labelled oriented tree $\Delta$, whose underlying graph is obtained by adding one edge and one vertex to $\Gamma^{\prime}$. By Proposition $4.2 P(\Gamma)$ is equivalent rel $P\left(\Gamma^{\prime}\right)$ to $P(\Delta)$. But $P(\Delta)$ is obtained from $P\left(\Gamma^{\prime}\right)$ by adding one generator $u$ and one relator $r$. Moreover $u$ appears with exponent sum $\mp 1$ in $r$, so in particular is properly involved in $r$. Hence $P(\Delta)$ reduces to $P\left(\Gamma^{\prime}\right)$, and the proof is complete.

8. Proofs of Theorems A and B. In this section we apply the results of the two preceding sections to prove Theorem B. Theorem A follows immediately from Theorem B and Fact 5.1, since ribbon disc complements are homology circles with 2-complex spines.

To prove Theorem B it is sufficient, by Proposition 3.2, to show that $G(\Gamma)$ is locally indicable for any labelled oriented tree $\Gamma$ of diameter at most 3. Consider first the case where $\Gamma$ has a proper admissible subtree $\Gamma^{\prime}$ such that each vertex of $\Gamma-\Gamma^{\prime}$ is extremal in $\Gamma$. By Corollary 7.2 the presentation $P(\Gamma)$ is equivalent rel $P\left(\Gamma^{\prime}\right)$ to a presentation which reduces to $P\left(\Gamma^{\prime}\right)$. By Fact 5.3 it is sufficient to prove that $G\left(\Gamma^{\prime}\right)$ is locally indicable.

By induction on the number of vertices of $\Gamma$, we may assume that $\Gamma$ is reduced, and that $\Gamma$ has no proper admissible subtree $\Gamma^{\prime}$ such that every vertex of $\Gamma-\Gamma^{\prime}$ is extremal in $\Gamma$. In particular this excludes the case of diameter 1 ( $\Gamma$ is not reduced), and the case of diameter 2 (take $\Gamma^{\prime}$ to be the subtree consisting of the unique nonextremal vertex). The case of diameter 0 is clearly trivial, so we an assume $\Gamma$ has diameter 3. Then $\Gamma$ has precisely two nonextremal vertices, say 0 and 1 (Figure 5). Since $\Gamma$ is reduced, each of the remaining vertices $2, \ldots, m$ occurs at least once as a label. There are precisely two possibilities (up to renumbering the vertices), since $|E(\Gamma)|=|V(\Gamma)|-1=m$. 


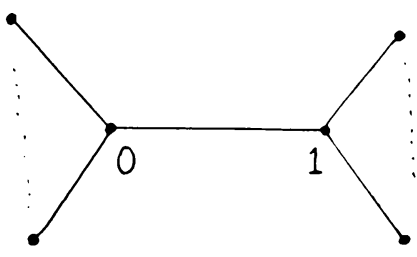

FiguRE 5

Case 1. Each of $1, \ldots, m$ occurs precisely once as a label.

Case 2. Each of $3, \ldots, m$ occurs precisely once as a label, while 2 occurs precisely twice.

Let $\Gamma^{\prime}$ denote the admissible subgraph of $\Gamma$ generated by $\{0,1\}$ in Case 1 , and by $\{0,1,2\}$ in Case 2. The restriction $\lambda \mid: E(\Gamma)-E\left(\Gamma^{\prime}\right) \rightarrow V(\Gamma)-V\left(\Gamma^{\prime}\right)$ of the labelling function $\lambda$ is injective (in Case 2 since $\Gamma^{\prime}$ must contain the two edges labelled 2) and surjective (since each vertex of $\Gamma-\Gamma^{\prime}$ occurs as a label). Hence $\chi\left(\Gamma^{\prime}\right)=\chi(\Gamma)$ $=1$, and $\Gamma^{\prime}$ is a tree. Since $\Gamma^{\prime}$ contains the two nonextremal vertices of $\Gamma$, we must have $\Gamma^{\prime}=\Gamma$ by our assumptions about $\Gamma$. Now apply Theorem 7.1 to $\Gamma$ and the admissible subgraph consisting only of the vertices 0 and 1 (Case 1), or 0,1 and 2 (Case 2).

In Case $1, \Gamma$ is equivalent to a weakly labelled oriented tree on 2 vertices, so $P(\Gamma)$ is equivalent to a one-relator (hence reducible) presentation, and $G(\Gamma)$ is locally indicable by Fact 5.2 (or directly by [4]).

In Case 2, $\Gamma$ is equivalent to a weakly labelled oriented tree on 3 vertices, in which the labels are both reduced sloping words. Then $G(\Gamma)$ is locally indicable by Theorem 6.2.

9. Some extensions to the case of diameter 4. A crucial factor in the proof of Theorem B is the fact that any tree of diameter 3 has precisely two nonextremal vertices. This is what enables us to reduce the problem to the study of a small presentation (at worst 2 relators), and Theorem 6.2 completes the job for us. If we consider trees of diameter greater than 3 , then we can no longer hope for results as general as Theorems A and B (at least with the methods of this paper). Even in the case of diameter 4 , there is no upper bound to the possible number of nonextremal vertices.

Nevertheless, the machinery developed in $\$ \S 6$ and 7 is sufficiently powerful to solve a number of special cases of the problem. In this section we state some results which extend Theorem B to suitable labelled oriented trees of diameter 4. For simplicity, we restrict attention to the case of a tree $\Gamma$ with precisely 3 nonextremal

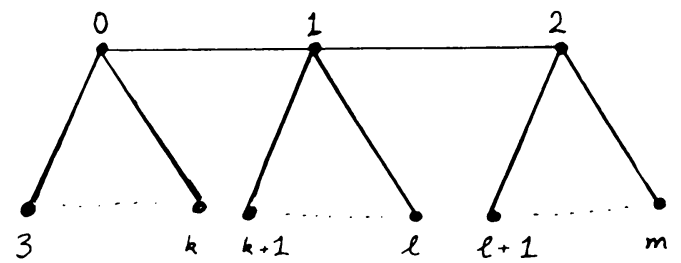

FIGURE 6 
vertices $0,1,2$ (Figure 6). We also assume that $\Gamma$ is reduced, that is that each of the extremal vertices $3, \ldots, m$ appears at least once as a label.

Proposition 9.1. If no vertex appears more than once as a label, then $G(\Gamma)$ is locally indicable.

Proposition 9.2. If one of $\{0,1,2\}$ appears twice as a label, then $G(\Gamma)$ is locally indicable.

Proposition 9.3. If $k=l$ and one of $\{0,1,2\}$ appears as a label, then $G(\Gamma)$ is locally indicable.

Proposition 9.4. If $k=l$ and some vertex appears three times as a label, then $G(\Gamma)$ is locally indicable.

The proofs are all variants of the proof of Theorem B. Consider the subgraph generated by $\{0,1,2\}$ for 9.1 and 9.2 , or by $\{0,2, j\}$ for 9.3 and 9.4 , where $j$ is the unique vertex which appears more than once as a label. In all cases, the proof reduces to consideration of a weakly labelled oriented tree on three vertices, and Theorem 6.2 applies.

10. Some further criteria for asphericity. Let $\Gamma$ be a labelled oriented graph, and consider the three maps $\iota, \tau, \lambda: E(\Gamma) \rightarrow V(\Gamma)$. The rôles played by $\iota$ and $\tau$ are similar, and interchanging them only amounts to giving $\Gamma$ the opposite orientation, which does not change the homotopy type of $K(\Gamma)$. On the other hand the labelling

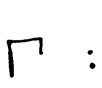

$I(\Gamma):$
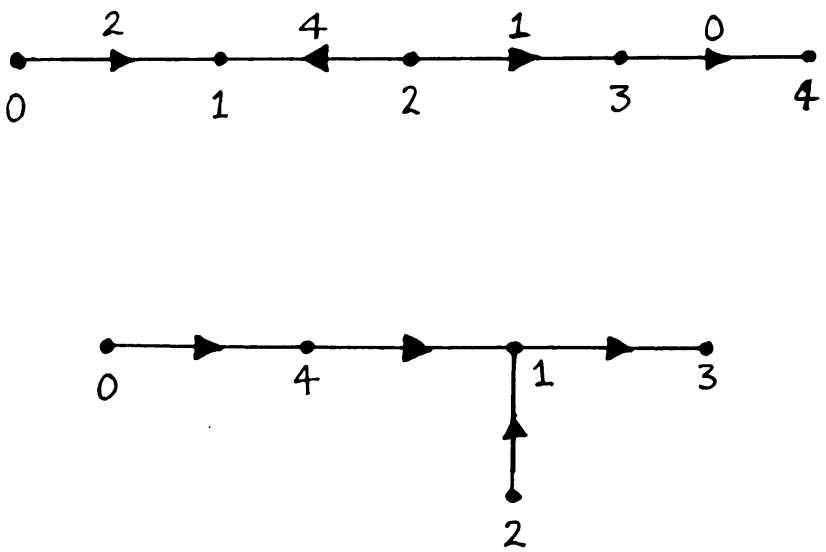

$T(r):$

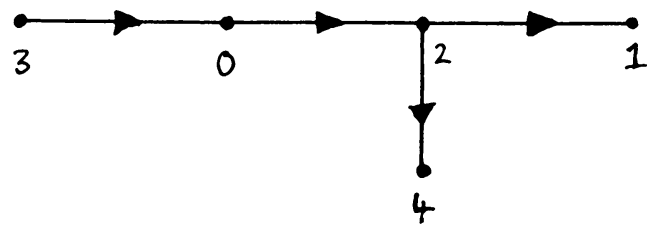

FIGURE 7 


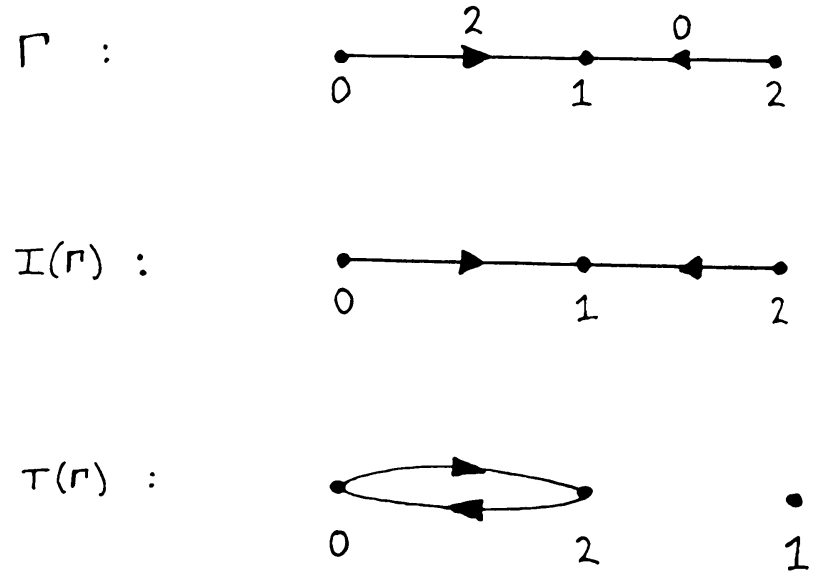

Figure 8

function $\lambda$ plays an entirely different rôle, so interchanging $\iota$ and $\lambda$ gives a labelled oriented graph $I(\Gamma)$ which is, in general, essentially different from $\Gamma$. We will not, in fact, be concerned with the labelling function $\iota$ of $I(\Gamma)$, merely with its underlying graph structure, with initial and terminal vertex maps $\lambda$ and $\tau$.
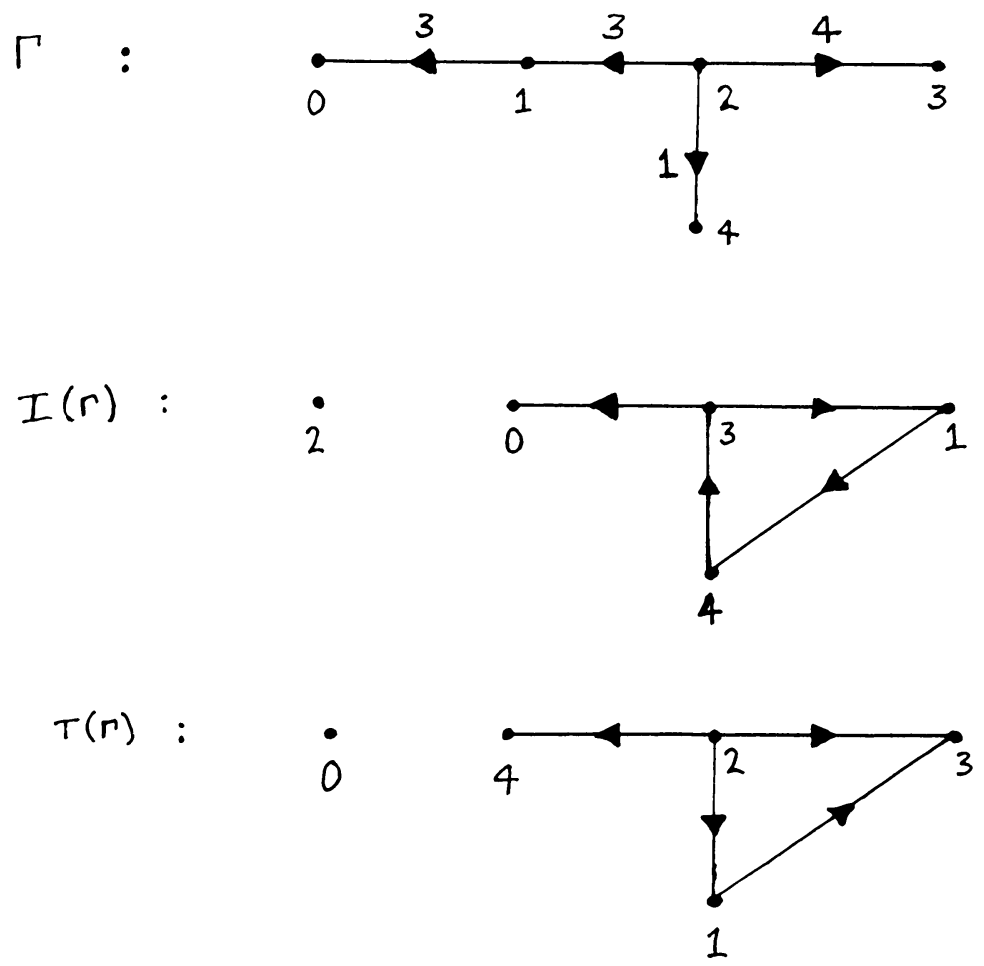

FI GURE 9 
Similarly, we may form a graph $T(\Gamma)$, with the same vertex and edge sets as $\Gamma$, and initial and terminal vertex maps $\iota$ and $\lambda$ respectively.

Consider the examples in Figures 7-9. In each case $\Gamma$ is a tree. In Figure $7 I(\Gamma)$ and $T(\Gamma)$ are also trees. In Figure $8 I(\Gamma)$ is a tree but not $T(\Gamma)$. In Figure 9 neither $I(\Gamma)$ nor $T(\Gamma)$ is a tree. These various possibilities are of interest because of the following result.

THEOREM 10.1. Let $\Gamma$ be a labelled oriented tree. If one of $I(\Gamma), T(\Gamma)$ is a tree, then the derived subgroup $G(\Gamma)^{\prime}$ of $G(\Gamma)$ is locally free (and so $G(\Gamma)$ is locally indicable). If both are trees, then $G(\Gamma)^{\prime}$ is free of rank $|E(\Gamma)|$.

Proof. Let $F$ be the free group on the generators $V(\Gamma)$ of $P(\Gamma)$. Pick a base point $t \in V(\Gamma)$. Let $F_{0}$ be the subgroup of $F$ generated by $\left\{u^{-1} v ; u, v \in V(\Gamma)\right\}$, and let $F_{1}$ be the subgroup generated by $\left\{u v^{-1} ; u, v \in V(\Gamma)\right\}$. Then $F$ is the free product $F_{0} *\langle t\rangle$ and $F_{1}=t F_{0} t^{-1}$. Since the image of $t$ generates $G(\Gamma) / G(\Gamma)^{\prime}$ and the image of $F_{0}$ lies in $G(\Gamma)^{\prime}$, it follows that $G(\Gamma)^{\prime}$ is the image in $G(\Gamma)$ of the normal closure of $F_{0}$.

For any edge $e$ of $\Gamma$, define $y_{e}=\lambda(e)^{-1} \iota(e) \in F_{0}, z_{e}^{\prime}=\tau(e) \lambda(e)^{-1} \in F_{1}$ and $z_{e}=t^{-1} z_{e}^{\prime} t \in F_{0}$. If $T(\Gamma)$ is a tree, then $\left\{y_{e} ; e \in E(\Gamma)\right\}$ is a basis for $F_{0}$, and the map $y_{e} \mapsto z_{e}$ defines an endomorphism $\theta$ of $F_{0}$. The relators of $P(\Gamma)$ can be rewritten

$$
\iota(e)^{-1} \lambda(e) \tau(e) \lambda(e)^{-1}=y_{e}^{-1} z_{e}^{\prime}=y_{e}^{-1} t z_{e} t^{-1}=y_{e}^{-1} t \theta\left(y_{e}\right) t^{-1} \quad(e \in E(\Gamma))
$$

and it follows that $G(\Gamma)^{\prime}$ is the direct limit of

$$
F_{0} \stackrel{\theta}{\rightarrow} F_{0} \stackrel{\theta}{\rightarrow} F_{0} \stackrel{\theta}{\rightarrow} \cdots
$$

and so locally free.

Similarly, if $I(\Gamma)$ is a tree, then $\left\{z_{e}^{\prime} ; e \in E(\Gamma)\right\}$ is a basis for $F_{1}$, so $\left\{z_{e} ; e \in E(\Gamma)\right\}$ is a basis for $F_{0}$. The map $z_{e} \mapsto y_{e}$ defines an endomorphism $\phi$ of $F_{0}$, and $G(\Gamma)^{\prime}$ is the direct limit of

$$
F_{0} \stackrel{\phi}{\rightarrow} F_{0} \stackrel{\phi}{\rightarrow} F_{0} \stackrel{\phi}{\rightarrow} \cdots \text {. }
$$

Finally, if both $I(\Gamma)$ and $T(\Gamma)$ are trees, then $\theta$ and $\phi$ are mutually inverse isomorphisms, and $G(\Gamma)^{\prime} \cong F_{0}$ is free of the stated rank.

COROllary 10.2. If either $I(\Gamma)$ or $T(\Gamma)$ is a tree, then $K(\Gamma)$ is aspherical.

Now let $U(\Gamma)$ be the graph formed from $I(\Gamma)$ and $T(\Gamma)$ by identifying their vertex sets. That is to say $U(\Gamma)$ has vertex set $V(\Gamma)$ and edge set $E(\Gamma) \times\{0,1\}$, with initial vertex map $(e, 0) \mapsto \lambda(e),(e, 1) \mapsto \iota(e)$ and terminal vertex map $(e, 0) \mapsto \tau(e)$, $(e, 1) \mapsto \lambda(e)$.

THEOREM 10.3. Let $\Gamma$ be a labelled oriented graph such that $U(\Gamma)$ contains no cycle of length 3 or less. Then $K(\Gamma)$ is aspherical, and $P(\Gamma)$ has soluble word and conjugacy problems.

Proof. That $U(\Gamma)$ contains no cycle of length 1 (that is, no loop) is just the statement that no edge of $\Gamma$ is labelled by one of its endpoints. Thus the defining relators of $P(\Gamma)$ are all cyclically reduced words of length 4 . 
Now there is an edge of $U(\Gamma)$ connecting two vertices $u, v$ for each relator in which $u^{\mp 1} v^{\mp 1}$ or $v^{\mp 1} u^{\mp 1}$ appears as a (cyclic) subword. Thus the statement that $U(\Gamma)$ contains no cycle of length 2 implies, in small cancellation terms, that no "piece" in $P(\Gamma)$ has length more than 1. In particular, $P(\Gamma)$ satisfies the small cancellation condition $C(4)$.

Similarly, the fact that $U(\Gamma)$ contains no cycle of length 3 implies that $P(\Gamma)$ satisfies the small cancellation condition $T(4)$. Hence $K(\Gamma)$ is aspherical, indeed $P(\Gamma)$ is diagramatically aspherical, in the sense of Chiswell, Collins and Huebschmann [5]. It also follows that $P(\Gamma)$ has soluble word and conjugacy problems. (For the necessary details of small cancellation theory, see for example Chapter V of [14].)

11. A class of aspherical 2-relator presentations. This final section was motivated by the following question which was put to me by Martin Dunwoody. Let $F$ be the free group with basis $\{x, y, z\}$, and let $f$ be the endomorphism of $F$ defined by $f(x)=x^{2} y x^{-1} y^{-1}, f(y)=y^{2} z y^{-1} z^{-1}, f(z)=z^{2} x z^{-1} x^{-1}$. Is the presentation

$$
P_{2}:\left\langle x, y, z \mid f^{2}(x), f^{2}(y)\right\rangle
$$

aspherical? Here $f^{2}(x)$ denotes $f(f(x))$, and so on. More generally, we will show that, for all $n \geqslant 0$, the group $G_{n}$ given by the presentation

$$
P_{n}:\left\langle x, y, z \mid f^{n}(x), f^{n}(y)\right\rangle
$$

is locally indicable, whence $P_{n}$ is aspherical by Fact 5.1. For $n=0$ all this is trivial, while for $n=1$ it follows from Fact 5.2 and the reducibility of $P_{1}$. Note that $P_{n}$ is a subpresentation of

$$
P_{n}^{\prime}:\left\langle x, y, z \mid f^{n}(x), f^{n}(y), f^{n}(z)\right\rangle .
$$

Now $P_{1}^{\prime}$ is a presentation of the trivial group [10], and hence so is $P_{n}^{\prime}$ for all $n$, by an easy inductive argument. In particular, the asphericity of $P_{n}$ is a test case for the Whitehead Conjecture. It is not known whether $P_{n}^{\prime}$ is Andrews-Curtis trivial (equivalent to the empty presentation) for $n \geqslant 1$. Indeed $P_{1}^{\prime}$ has been proposed as a candidate for a counterexample to the Andrews-Curtis Conjecture [19]. Nevertheless we may still embed $P_{n}$ as a subpresentation in an Andrews-Curtis trivial presentation

$$
P_{n}^{\prime \prime}:\left\langle x, y, z \mid f^{n}(x), f^{n}(y), z\right\rangle,
$$

so the 2-complex associated to $P_{n}$ 3-deforms to a spine of some ribbon disc complement $X_{n}$ by [12, Theorem 4.2]. But $X_{n}$ does not come under the scope of Theorem A, since its dual graph $\Gamma_{n}$ will have large diameter in general. For example, we may take $\Gamma_{1}$ to be the labelled oriented chain of length 10 in Figure 10. Note that $\Gamma_{1}$ has an admissible subtree (Figure 11) corresponding to the subpresentation $\langle y, z \mid f(y)\rangle$ of $P_{1}$. The results of $\S 10$ do not apply here either, since neither $I\left(\Gamma_{1}\right)$ nor $T\left(\Gamma_{1}\right)$ is a tree, and $U\left(\Gamma_{1}\right)$ contains cycles of length 2 . Of course, none of our results rule out the possibility that $G_{1} \cong G\left(\Gamma^{\prime}\right)$ for some other labelled oriented tree $\Gamma^{\prime}$ to which some of the results of previous sections apply. This seems unlikely, however.

We now construct a sequence of presentations $Q_{n}(n \geqslant 0)$ as follows. Define

$$
Q_{0}=\left\langle x_{0}, y_{0}, z_{0} \mid x_{0}, y_{0}\right\rangle \text {. }
$$




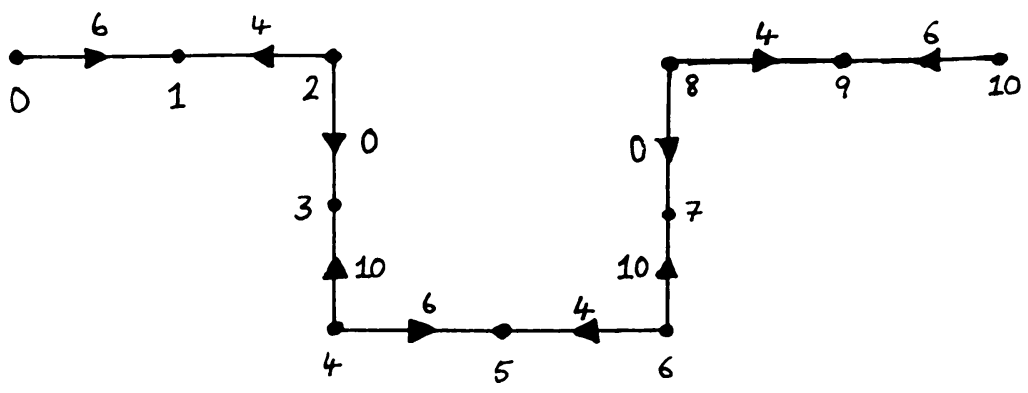

Figure 10

Inductively, define $Q_{n}$ by adding generators $x_{n}, y_{n}, z_{n}$ and relators $x_{n-1}^{-1} x_{n}^{2} y_{n} x_{n}^{-1} y_{n}^{-1}$, $y_{n-1}^{-1} y_{n}^{2} z_{n} y_{n}^{-1} z_{n}^{-1}, z_{n-1}^{-1} z_{n}^{2} x_{n} z_{n}^{-1} x_{n}^{-1}$ to $Q_{n-1}$ for $n \geqslant 1$. Clearly $Q_{n}$ is equivalent to $P_{n}$ for all $n$.

Furthermore, by adding the relator $z_{n}$ to $Q_{n}$, we obtain a presentation $Q_{n}^{\prime}$ equivalent to $P_{n}^{\prime \prime}$. An easy inductive argument shows that $Q_{n}^{\prime}$ is Andrews-Curtis trivial, whence $P_{n}^{\prime \prime}$ is Andrews-Curtis trivial, as claimed above.

Let $Y_{n}$ be the 2-complex corresponding to $Q_{n}$, and let $\tilde{Y}_{n}$ be its maximal abelian cover.

THEOREM 11.1. $\tilde{Y}_{n}$ is reducible for all $n$.

COROLlaRY 11.2. $G_{n}$ is locally indicable, and $Y_{n}$ is aspherical.

Proof of TheOrem 11.1. The proof is by induction on $n$, the case $n=0$ being trivial. Suppose $n \geqslant 1$. Then $Y_{n}$ is obtained from $Y_{n-1}$ by adding 1-cells $a, b, c$ (corresponding to the generators $x_{n}, y_{n}, z_{n}$ of $P_{n}$ ), and 2-cells $\alpha, \beta, \gamma$. The 2-cells are attached via the paths

$$
\alpha: u^{-1} a^{2} b a^{-1} b^{-1}, \quad \beta: v^{-1} b^{2} c b^{-1} c^{-1}, \quad \gamma: w^{-1} c^{2} a c^{-1} a^{-1},
$$

respectively, where $u, v, w$ are the 1-cells of $Y_{n-1}$ corresponding to the generators $x_{n-1}, y_{n-1}, z_{n-1}$, respectively.

Since the inclusion $Y_{n-1} \subset Y_{n}$ induces an isomorphism $H_{1}\left(Y_{n-1}\right) \cong H_{1}\left(Y_{n}\right) \cong \mathbf{Z}$, the covering of $Y_{n-1}$ induced by $\tilde{Y}_{n}$ is just the maximal abelian covering $\tilde{Y}_{n-1}$, which is reducible by inductive hypothesis. Also $\tilde{Y}_{n}$ is obtained from $\tilde{Y}_{n-1}$ by adding 1-cells $a_{i}, b_{i}, c_{i}(i \in \mathbf{Z})$ and 2-cells $\boldsymbol{\alpha}_{i}, \beta_{i}, \gamma_{i}(i \in \mathbf{Z})$. The 2-cells are attached via the paths

$$
\alpha_{i}: u_{i}^{-1} a_{i}^{2} b_{i} a_{i}^{-1} b_{i}^{-1}, \quad \beta_{i}: v_{i}^{-1} b_{i}^{2} c_{i} b_{i+1}^{-1} c_{i}^{-1}, \quad \gamma_{i}: w_{i-1}^{-1} c_{i-1} c_{i} a_{i+1} c_{i}^{-1} a_{i}^{-1},
$$

respectively.

Let $Z$ be a finite subcomplex of $\tilde{Y}_{n}$ of dimension at least 1 . If $Z \subset \tilde{Y}_{n-1} \cup \tilde{Y}_{n}^{(1)}$, then there is an elementary reduction $\left(Z, Z^{\prime}\right)$, so suppose $Z$ contains at least one 2-cell not in $\tilde{Y}_{n-1}$. Let $s$ be the least integer such that one of $\alpha_{s}, \beta_{s}, \gamma_{s}$ belongs to $Z$. If $\gamma_{s} \in Z$, then $\left(Z, Z-\left\{c_{s-1}, \gamma_{s}\right\}\right)$ is an elementary reduction. If $\gamma_{s} \notin Z$ but $\alpha_{s} \in Z$, then $\left(Z, Z-\left\{a_{s}, \alpha_{s}\right\}\right)$ is an elementary reduction. If $\alpha_{s}, \gamma_{s} \notin Z$, then $\beta_{s} \in Z$ and $\left(Z, Z-\left\{b_{s}, \beta_{s}\right\}\right)$, is an elementary reduction. 


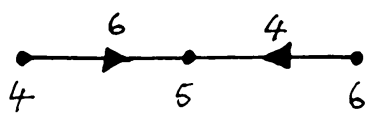

FIgURE 11

Hence $\tilde{Y}_{n}$ is reducible, as claimed.

Proof of Corollary 11.2. Apply Fact 5.2 and the theorem to see that $G_{n}^{\prime}$ is locally indicable. Then $G_{n}$ is also locally indicable, since $G_{n} / G_{n}^{\prime} \cong \mathbf{Z}$. Hence $Y_{n}$ is aspherical by Fact 5.1, since $H_{2}\left(Y_{n}\right)=0$.

\section{REFERENCES}

1. J. F. Adams, $A$ new proof of a theorem of W. H. Cockcroft. J. London Math. Soc. 49 (1955), 482-488.

2. J. J. Andrews and M. L. Curtis, Free groups and handlebodies, Proc. Amer. Math. Soc. 16 (1965), $192-195$.

3. K. Asano, Y. Marumoto and T. Yanagawa, Ribbon knots and ribbon discs, Osaka J. Math. 18 (1981), $161-174$.

4. S. D. Brodskiī, Equations over groups and groups with a single defining relation, Russian Math. Surveys 35 (1980), no. 4, 165.

5. I. M. Chiswell, D. J. Collins and J. Huebschmann, Aspherical group presentations, Math. Z. 178 (1981), 1-36.

6. T. Cochran, Ribbon knots in $S^{4}$, J. London Math. Soc. 28 (1983), 563-576.

7. S. Gersten, Conservative groups, indicability and a conjecture of Howie. J. Pure Appl. Algebra 29 (1983), 59-74.

8. C. McA. Gordon, Ribbon concordance of knots in the 3-sphere, Math. Ann. 257 (1981), 157-170.

9. J. Hass, The geometry of the slice-ribbon problem, Math. Proc. Cambridge Philos. Soc. 94 (1983), $101-108$.

10. G. Higman, A finitely generated infinite simple group, J. London Math. Soc. 26 (1951), 61-64

11. J. Howie, On locally indicable groups, Math. Z. 180 (1982), 445-461.

12. Some remarks on a problem of J. H. C. Whitehead. Topology 22 (1983), 475-485.

13. J. Howie and H. R. Schneebeli, Homological and topological properties of locally indicable groups, Manuscripta Math. 44 (1983), 71-93.

14. R. C. Lyndon and P. E. Schupp, Combinatorial group theory, Ergebnisse der Mathematik und ihrer Grenzgebiete, Band 89, Springer-Verlag, Berlin and New York, 1977.

15. S. J. Pride, Some finitely presented groups of cohomological dimension two with propert! (FA), J. Pure Appl. Algebra 29 (1983), 167-168.

16. P. Shalen, Infinitely divisible elements in 3-manifold groups, Ann. of Math. Studies, no. 84, Princeton Univ. Press, Princeton, N. J., 1975, pp. 293-335.

17. A. J. Sieradski, Combinatorial isomorphisms and combinatorial homotopy equiealences, J. Pure Appl. Algebra 7 (1976), 59-65.

18. J. Stallings, Surfaces in 3-manifolds and nonsingular equations in groups, Math. Z. 184 (1983), 1-17.

19. S. Young, Contractible 2-complexes, M. Sc. Dissertation, Cambridge, 1976.

Department of Mathematics, University of Glasgow, University Gardens. Glasgow G12 BQW. SCOTI.AND 